Mots. Les langages du politique

$80 \mid 2006$

La politique mise au net

\title{
L'épargne salariale en France dans deux discours politiques de gauche et de droite
}

\section{Stéphane Trébucq}

\section{(2) OpenEdition \\ Journals}

Édition électronique

URL : https://journals.openedition.org/mots/573

DOI : $10.4000 /$ mots.573

ISSN : 1960-6001

Éditeur

ENS Éditions

\section{Édition imprimée}

Date de publication : 1 mars 2006

Pagination : 105-119

ISBN : 2-84788-095-X

ISSN : 0243-6450

Référence électronique

Stéphane Trébucq, «L'épargne salariale en France dans deux discours politiques de gauche et de droite ", Mots. Les langages du politique [En ligne], 80 | 2006, mis en ligne le 01 mars 2008, consulté le 23 avril 2022. URL : http://journals.openedition.org/mots/573 ; DOl : https://doi.org/10.4000/mots. 573

\section{(C) ENS Éditions}




\section{L'épargne salariale en France dans deux discours politiques de gauche et de droite ${ }^{1}$}

De nombreuses modalités de gestion des entreprises font aujourd'hui encore l'objet de règlementations et de régulations à un échelon national. De ce fait, lors de leur élaboration, elles n'échappent pas au débat politique et à d'éventuelles controverses idéologiques. Il en va ainsi de l'intérêt que peut représenter éventuellement la détention du capital de l'entreprise par ses salariés, même si celle-ci n'est que partielle. En France, l’idée gaulliste de participation demeure un projet de société encore peu développé. En effet, il s'agit pour l'heure d'une réalité concernant essentiellement les grands groupes internationaux ${ }^{2}$. Partant, on observe l'expression d'un clivage gauche/droite ${ }^{3}$ quant à l'opportunité et aux modalités de l'extension de tels dispositifs. Alors qu'Édouard Balladur exerçait les fonctions de Premier ministre, un projet de loi visant à garantir aux salariés la détention d'un pourcentage des augmentations de capital de leur entreprise fut rejeté à l’Assemblée nationale. En 1999, le débat fut relancé par le sénateur de droite Jean Chérioux, qui produisit un rapport intitulé: «L'actionnariat salarié: vers un véritable partenariat dans l'entreprise ». Désireux de reprendre la main, le gouvernement Jospin a alors souhaité proposer son propre projet de loi. Pour ce faire, il s'est appuyé sur le rapport rédigé par Jean-Pierre Balligand et Jean-Baptiste de Foucault, consacré à l'épargne salariale. Celui-ci a été remis au chef du gouvernement en janvier 2000.

À ce document se sont ajoutés plusieurs rapports complémentaires émanant de différents organismes tels que le Parti socialiste, la chambre de commerce et d'industrie de Paris et le MEDEF. Parallèlement, la question de l'actionnariat salarié n'a pas été sans susciter le plus vif intérêt chez les partenaires sociaux. La remise en cause d'une série d'équilibres économiques, sociaux et

1. Cet article a été rédigé à partir de deux discours prononcés respectivement, en novembre 2000 , par Laurent Fabius, alors ministre des Finances du gouvernement socialiste de cohabitation dirigé par Lionel Jospin, et Christian Poncelet, porté à la présidence du Sénat par la majorité de droite de cette même assemblée.

2. En effet, seuls $2,5 \%$ des salariés des entreprises françaises de moins de 50 salariés disposent d'un plan d'intéressement.

3. M. Crapez, 1998, "De quand date le clivage gauche/droite en France? », Revue française de science politique, $\mathrm{n}^{\circ} 48$, février, p. $42-75$.

Université Montesquieu-Bordeaux4, trebucq@u-bordeaux4.fr 
politiques de la société n'y est probablement pas étrangère. D'aucuns n'ontils pas longtemps considéré qu'une convergence des intérêts entre les actionnaires et les salariés ne pouvait constituer qu'un rapprochement contre nature et, partant, préjudiciable à la cause des salariés? Il semble cependant que les oppositions se soient progressivement atténuées et que l'on se soit acheminé vers un large consensus. Le Parti communiste a ainsi voté le projet de loi sur l'épargne salariale, et la CGT n'est plus opposée à une généralisation de ce type de formule. En revanche, selon le témoignage du député Balligand, d'âpres discussions ont accompagné la définition des finalités et des modalités pratiques du projet de loi. Mais le contraire n'eût pas manqué de surprendre.

\section{Cadre de l'analyse}

Cette loi étant désormais en application, il n'est pas dénué d'intérêt de tester, a posteriori, les convergences et les divergences sur le sujet à partir des référents mobilisés par des hommes politiques. Le forum annuel organisé par Fondact ${ }^{4} \mathrm{le}$ 23 novembre 2000, à l'Automobile Club de Paris, nous a fourni l'occasion de réaliser une analyse de contenu comparative du discours gouvernemental tenu par le ministre de l'Économie, des Finances et de l'Industrie, Laurent Fabius, et du discours d'opposition prononcé par le président du Sénat, Christian Poncelet.

Dans un tel contexte, il va de soi que les prises de parole et de position sont révélatrices et engagent leurs auteurs. Elles s'adressent, en effet, à un public de chefs d'entreprises et de responsables de banques, mais aussi de représentants des salariés. D'autres acteurs sociaux se trouvent également concernés. Parmi ceux-ci figurent notamment les représentants des différents ministères, des organismes de conseil, d'institutions telles que la COB, la Banque de France ou la Caisse des Dépôts, la presse économique et des chercheurs en sciences sociales. Le discours de Laurent Fabius a été formulé en introduction à la journée organisée par Fondact. Christian Poncelet s'est exprimé, quant à lui, lors du déjeuner. Ces deux temporalités ont leur importance, le premier introduisant le débat en venant exposer l'action gouvernementale, le second venant la commenter. Ces deux discours n'étaient pas improvisés. Il s'agissait de productions écrites qui ont été, après leur lecture, distribuées au public. On peut donc considérer que ces deux hommes politiques ont pris soin de structurer leurs interventions en connaissance de cause, afin de convaincre au mieux leur auditoire. Naturellement, l'un et l'autre n'occupent pas la même fonction au regard de ce

4. Fondact est une association loi 1901 agissant en faveur de la gestion participative, de l'épargne salariale et de l'actionnariat de responsabilité. Elle a été fondée en 1980 par Rémy Schlumberger et forme un groupe organisé souhaitant promouvoir l'idée de participation. Elle regroupe à cet effet une centaine de sociétés françaises de diverses tailles, des sociétés de gestion et des cabinets de conseil, des syndicats et des associations d'actionnaires salariés. 
projet, et si le premier est à l'origine de cette loi, le second se situe lisiblement dans une démarche d'opposition, et plus généralement critique vis-à-vis de la politique menée par la gauche. L'analyse des référents mobilisés peut ainsi permettre de saisir objectivement les points communs et les différences observables dans leur représentation de cette thématique politico-économique. C'est dans cette double approche que réside l'intérêt de l'étude effectuée.

\section{Méthode d’analyse}

Les deux textes ont été analysés à l'aide du logiciel d'analyse de contenu Tropes. Dans cette démarche, l'unité choisie pour le découpage des allocutions est la proposition grammaticale (sujet-verbe-complément), conformément à l'analyse propositionnelle de discours développée notamment par Rodolphe Ghiglione ${ }^{5}$. Après ce découpage propositionnel, les deux textes ont été analysés sur un plan sémantique. Les fréquences d'occurrences des « références utilisées » peuvent ainsi être relevées, de même que la mise en relation de ces références au sein d'une même proposition. Le niveau d'agrégation sémantique des termes est ici le plus faible, de telle sorte que les champs sémantiques isolés dans le texte contiennent le plus petit nombre de termes ${ }^{6}$. De la sorte, les références communes aux deux discours, ainsi que les références spécifiques et distinctives, ont pu être identifiées. La mise en relation des références permet également de proposer une représentation des principales cooccurrences du discours, approchant la notion d'association d'idées ou de concepts. En outre, une série de dénombrements statistiques portant sur les occurrences des formes grammaticales des textes peut être établie à l'aide du logiciel, dévoilant les stratégies mises en œuvre par les deux hommes politiques. Enfin, les propositions remarquables sont recensées grâce à l'algorithme de sélection présent dans le logiciel, dont la méthodologie est mal explicitée. Il s'agit de propositions supposées essentielles, et dites « représentatives » de la structure fondamentale du texte.

\section{Résultats de l’analyse}

Les discours politiques correspondent à un mode de communication très fortement contraint. Il s'agit généralement de transmettre un message donné dans

5. R. Ghiglione, B. Matalon, N. Bacri, 1985, Les dires analysés. L'analyse propositionnelle du discours, Saint-Denis, Presses universitaires de Vincennes, Centre de recherche de l'université de Paris 8.

6. Les regroupements de termes en champs sémantiques sont à ce niveau préchargés dans le logiciel Tropes. Ils peuvent apparaitre, de ce point de vue, discutables. Il revient alors à l'analyste de vérifier la qualité et la fiabilité de ces regroupements en fonction des textes étudiés. 
un temps limité, tout en réussissant à mobiliser la terminologie et les raisonnements entrant en adéquation avec la pensée politique du groupe dont se réclame l'orateur. Le contenu des textes devient alors particulièrement révélateur des intentions animant leurs auteurs.

\section{Les thèmes du discours}

Une première observation permet de constater une différence évidente quant à la longueur des discours respectifs. Laurent Fabius réalise une intervention relativement brève, composée de 91 propositions et de 835 mots, soit une moyenne de 9,17 mots par proposition, « ce qui correspond à des propositions syntaxiquement simples ${ }^{7}$, aisément assimilables. L'intervention de Christian Poncelet est plus longue, le texte étant composé de 236 propositions et de 2023 mots, mais avec un nombre de mots par proposition plus réduit $(8,57)$. Ce discours s'avère plus structuré et mobilise un nombre de références plus important.

La sélection des cinq références les plus utilisées dans chaque texte (voir tableau 1) conduit à retenir une fréquence d'occurrences supérieure à o,49 \% ${ }^{8}$. Leur comparaison constitue une première approche des différences de représentations entre les deux discours.

\begin{tabular}{|l|c|l|c|}
\hline \multicolumn{2}{|c|}{ Texte de Laurent Fabius } & \multicolumn{2}{c|}{ Texte de Christian Poncelet } \\
\hline $\begin{array}{l}\text { Références utilisées } \\
\text { ou notions }\end{array}$ & $\begin{array}{c}\text { Fréquence } \\
\text { relative }\end{array}$ & $\begin{array}{l}\text { Références utilisées } \\
\text { ou notions }\end{array}$ & $\begin{array}{c}\text { Fréquence } \\
\text { relative }\end{array}$ \\
\hline Entreprise & $1,44 \%$ & Épargne & $1,09 \%$ \\
\hline Salarié (employé) & $1,32 \%$ & Économie & $0,59 \%$ \\
\hline Épargne & $0,96 \%$ & Actionnariat & $0,54 \%$ \\
\hline Économie & $0,60 \%$ & Entreprise & $0,54 \%$ \\
\hline Travail (emploi) & $0,60 \%$ & Retraite & $0,49 \%$ \\
\hline
\end{tabular}

Tableau 1. Références utilisées

Ce tableau comparatif simplifié, ne retenant que les cinq premières références avec les plus fortes fréquences, met en évidence une hiérarchisation différente des aspects importants du sujet traité. Pour Laurent Fabius, la relation entre l'entreprise et le salarié est centrale. Elle permet de déboucher sur une épargne favorable à la fois à l'économie et à l'emploi. En revanche, pour Christian

7. R. Ghiglione et al., ibid., p. 101.

8. Ce pourcentage correspond au nombre d'occurrences de la référence utilisée divisé par le nombre de mots présents dans le texte. Rappelons que les « références utilisées » sont établies par la projection sur les textes d'une grille sémantique reposant sur un dictionnaire préétabli. 
Poncelet, il s'agit avant tout d'un problème économique d'épargne. À ses yeux, le développement de l'actionnariat salarié au sein de l'entreprise doit pouvoir apporter une réponse au problème des retraites.

Afin de compléter cette première phase, nous avons distingué, d'une part, les objets communs aux deux discours, et d'autre part, ceux qui les différencient (voir tableau 2).

\begin{tabular}{|l|l|}
\hline $\begin{array}{l}\text { Références ou notions communes } \\
\text { présentant plus de trois occurrences }\end{array}$ & $\begin{array}{l}\text { An (Durée), Changement, Économie, } \\
\text { Entreprise, Épargne, Loi, Salarié, Travail }\end{array}$ \\
\hline $\begin{array}{l}\text { Références ou notions distinctives } \\
\text { utilisées dans le discours de Laurent } \\
\text { Fabius }\end{array}$ & Accord, Dirigeant, Investissement \\
\hline $\begin{array}{l}\text { Références ou notions distinctives } \\
\text { utilisées dans le discours de Christian } \\
\text { Poncelet }\end{array}$ & $\begin{array}{l}\text { Actionnariat salarié, Pensée, } \\
\text { Question, Problème, Rapport }\end{array}$ \\
\hline
\end{tabular}

Tableau 2. Références communes et spécifiques aux deux hommes politiques

Les références, permettant de modéliser la question, diffèrent tout d'abord dans l'usage qu'ils font des divers vocables: Laurent Fabius parle d'épargne salariale, tandis que Christian Poncelet évoque plus volontiers l'actionnariat salarié. Cependant, les deux discours se rejoignent à propos du changement économique que ne va pas manquer d'engendrer la nouvelle loi, notamment quant au travail du salarié mais aussi quant à son épargne investie au sein de l'entreprise.

Cependant, Laurent Fabius insiste plus particulièrement sur les conditions qui doivent présider à la mise en œuvre de la loi. Celle-ci prendra appui sur un accord collectif au niveau « d'une branche professionnelle, d'un territoire ou d'un groupe d'entreprises ». Il évoque en outre une catégorie d'acteurs non abordée par Christian Poncelet, à savoir celle des employeurs ou des dirigeants. Ces allusions s'avèrent plutôt péjoratives puisque, selon le ministre, les réseaux de collecte et la mutualisation des placements au niveau des PME présentera l'avantage d' ' éviter le tête-à-tête financier entre employé et employeur ». Il ajoute que le salarié verra la continuité de ses droits garantie lors d'un éventuel «changement d'employeur ». Le discours de Laurent Fabius se range ainsi, résolument, du côté de la cause des salariés. Toutefois, cette position se trouve contrebalancée par la présentation d'une disposition prise en faveur des dirigeants et des mandataires sociaux; la loi, en effet, prévoit que ces derniers puissent bénéficier des plans d'épargne au sein des entreprises de moins de 100 salariés. Par ailleurs, le ministre de l'Économie souligne, à deux reprises, la notion d'investissement, celle-ci étant considérée comme une variable économique déterminante. Le terme retraite n'est utilisé qu'une seule fois dans le texte de Laurent 
Fabius, alors qu'il ne figure pas moins de dix fois dans le texte de Christian Poncelet. Ce dernier accorde ainsi une place centrale au problème des retraites, là où le gouvernement a souhaité très nettement distinguer le projet d'épargne salariale de la création, sous une forme plus ou moins détournée ${ }^{9}$, de fonds de pension.

Christian Poncelet relève l'intérêt grandissant suscité par l'actionnariat salarié. Il n'en veut pour preuve que l'abondance des rapports auxquels cela a donné lieu. Cependant, il s'interroge à plus d'un titre quant à la cohérence de l'action gouvernementale. Le vocable salaire n'est alors utilisé qu'une seule fois dans le texte de Christian Poncelet. En revanche, ce dernier souligne bien la question des retraites constamment éludée par le pouvoir socialiste. Le discours de Christian Poncelet étant par ailleurs plus étoffé que celui de Laurent Fabius, cela lui donne l'opportunité de mobiliser un plus grand nombre de références telles que: stratégie, inégalités, fonds de pension, volonté, concertation, entrepreneur, signe. Il utilise aussi l'expression percutante de stratégie du crabe, empruntée à Éric Israëlevitch, faisant probablement référence au forum de l'année précédente, durant lequel cet auteur avait été cité. En définitive, le président du Sénat tient surtout à dénoncer l'attitude ambigüe du gouvernement en place, consistant, « lorsqu'un obstacle idéologique est trop important, à le contourner, si possible en proclamant que l'on fait le contraire ».

Autre opposition flagrante: Laurent Fabius avance la mise en place d'une économie solidaire, tandis que Christian Poncelet fustige les fauteurs d'inégalités. C'est ainsi qu'il déclare: «Pire, au lieu de favoriser les valeurs en lesquelles ils croient, ils les trahissent et font le lit de toutes les inégalités ${ }^{10}$ : inégalités entre fonctionnaires et salariés; inégalités entre salariés des grandes entreprises et ceux des PME; inégalités entre ceux qui ont une capacité d'épargner et ceux qui ne l'ont pas. » Le président du Sénat marque ainsi tout l'intérêt qu'il porte à l'égalité de traitement de l'ensemble des Français. Il en profite pour pointer le paradoxe du conservatisme gouvernemental, conduisant notamment à surseoir, pour des raisons électoralistes, à la résolution du problème des retraites. En tout état de cause, Christian Poncelet considère que la volonté du gouvernement n'apparait pas clairement. À ce propos, il relève des contradictions flagrantes entre la position de l'ancien ministre de l'Économie, Dominique Strauss-Kahn, souhaitant placer «l'épargne salariale au cœur du projet socialiste » et "infléchir l'évolution du capitalisme», et le contenu du rapport Balligand-de Foucault ayant inspiré la loi Fabius, selon lequel « ce serait une erreur que de croire que l'épargne salariale permettrait de résoudre l'anta-

9. La période d'épargne de dix ans et la sortie du produit de placement sous forme de capital ont permis de différencier, dans l'esprit de l'opinion publique, le projet de loi d'un système de fonds de pension disposant habituellement d'une période de placement plus longue et d'une sortie sous forme de rente. Cette distinction reste cependant purement formelle, certains organismes privés étant parfaitement en mesure d'assurer l'allongement des placements et la conversion en rente d'une sortie fixée initialement en capital.

10. Les termes en italique dans cette citation figurent en caractère gras dans le texte original. 
gonisme naturel entre le capital et le travail ». Christian Poncelet regrette également l'utilisation dévoyée de la concertation par le gouvernement, masquant, selon lui, «l'attentisme et l'immobilisme». En ce sens, la politique actuellement menée est un « signe de démagogie ».

Si cette première analyse des contenus a permis de mieux appréhender le clivage existant entre les deux discours prononcés devant le public du forum Fondact, une étude plus approfondie peut être proposée à partir de la mise en relation des références utilisées et des statistiques tirées des textes, au moyen du logiciel Tropes.

\section{La représentation du problème}

La mise en relation des références, ou cooccurrences, utilisées par Laurent Fabius offre l'opportunité d'élaborer la carte, présentée ci-après, mobilisant les notions de salarié, d'entreprise, d'épargne et de dirigeant. Les flèches établissent les relations actant-acté ${ }^{11}$ liant entre elles les références utilisées. Les flèches les plus épaisses signalent les relations disposant d'une fréquence supérieure.

\begin{tabular}{|l|c|}
\hline \multicolumn{1}{|c|}{ Mises en relation } & Nombre de relations observées \\
\hline salarié ---> entreprise & 3 \\
\hline épargne ---> salarié & 2 \\
\hline dirigeant ---> épargne & 2 \\
\hline
\end{tabular}

Tableau 3. Mises en relation des références du discours de Laurent Fabius

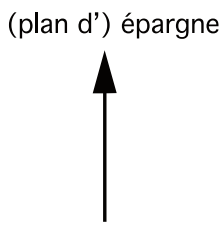

dirigeant

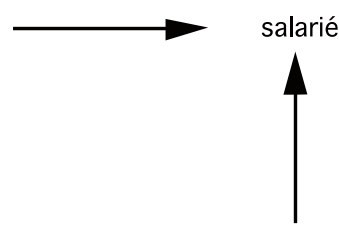

entreprise

Graph. 1. Carte des mises en relation de références du discours de Laurent Fabius

11. Un terme est en position d'actant par rapport à un autre terme considéré comme acté lorsque le premier (actant) précède le second (acté). Par exemple, dans la phrase «Changements d'employeur et de plan d'épargne seront neutres au regard des droits du salarié », la référence au plan d'épargne est en position d'actant, et celle de salarié en position d'acté. Le logiciel Tropes sélectionne l'association des deux références lorsque cette même situation se reproduit au moins deux fois dans les propositions du corpus étudié. On résume alors la situation de la façon suivante: plan d'épargne salarié. 
On obtient, en revanche, un tableau des mises en relation et une cartographie très différents pour le président du Sénat, Christian Poncelet.

\begin{tabular}{|l|c|}
\hline \multicolumn{1}{|c|}{ Mises en relation } & Nombre de relations observées \\
\hline épargne ---> retraite & 4 \\
\hline instrument ---> épargne & 4 \\
\hline problème ---> retraite & 3 \\
\hline loi ---> épargne & 2 \\
\hline épargne ---> actionnariat & 2 \\
\hline capital --> travail & 2 \\
\hline
\end{tabular}

Tableau 4. Mises en relation des références du discours de Christian Poncelet

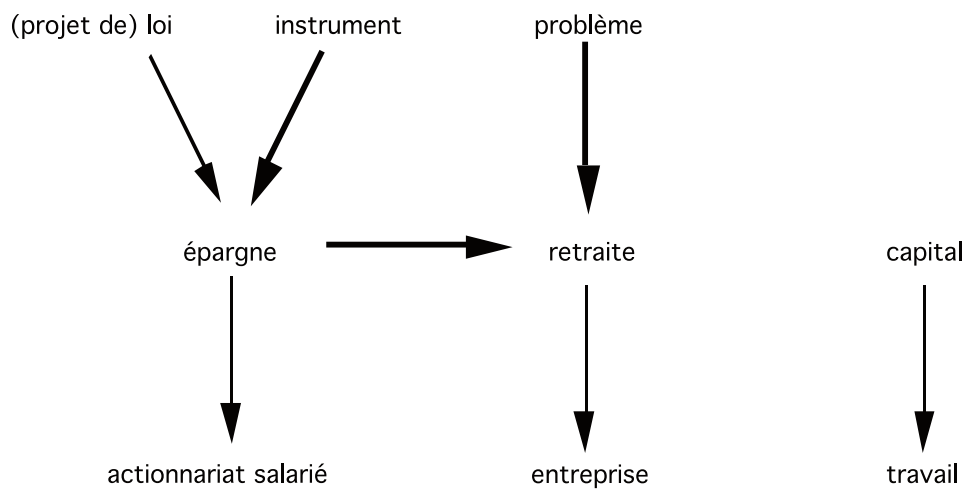

Graph. 2. Carte des mises en relation des références du discours de Christian Poncelet

La lecture des deux cartes ainsi obtenues saisit au mieux les différences de représentation existant entre Laurent Fabius et Christian Poncelet à propos de l'épargne salariale.

La présentation du ministre de l'Économie fait apparaitre une relation entre le salarié et l'entreprise, le plan d'épargne et le salarié, ainsi qu'entre le dirigeant et le plan d'épargne. Ces mises en relation découlent certainement de la nécessité d'une présentation technique des dispositions du projet de loi, mais aussi, et surtout, de l'approche de la question qui a été retenue et qui a conduit ainsi à l'élaboration d'un tel projet de loi.

En ce qui le concerne, le président du Sénat propose une approche alternative conduisant à mettre en relation, prioritairement, les « instruments d'épargne salariale » et le «problème des retraites », puis «l'épargne » et "l'actionnariat salarié », et finalement le «capital» et le «travail». Notons que cette 
dernière relation inscrit le discours de Christian Poncelet dans la lignée du courant « utopiste » représenté par Charles Fourier, Louis Blanc, voire Pierre-Joseph Proudhon, qui ont tenté de remettre en question et d'annuler l'opposition traditionnelle travail-capital.

Le discours de Laurent Fabius, en contraste, s'appuie sur un courant d'une sensibilité plus «productiviste », chère aux saint-simoniens, qui fait de la participation aux résultats et de l'association au capital un facteur d'amélioration, quantitative et qualitative, de la performance par la motivation des salariés. Cet élément corrobore l'appréciation portée par Gérard Hirigoyen en 1997, selon lequel « la conception productiviste l'emporte définitivement, au moins dans l'esprit des pouvoirs publics », depuis 1981 . Toutefois, on peut relever aussi dans le discours de Christian Poncelet la mention de l'intérêt de l'actionnariat salarié, selon une logique productiviste, et instrumentale, compatible avec la volonté du dirigeant de se maintenir en poste. En revanche, aucun des deux discours ne semble se référer à un troisième courant de pensée dit « humaniste », selon lequel l'actionnariat salarié serait susceptible d'assurer la dignité de l'homme au travail.

\section{La stratégie du discours}

L'examen des statistiques obtenues au moyen du logiciel Tropes (voir tableau 3) permet une approche plus fine des différences de structure affectant des textes mis au service d'une stratégie politique.

La comparaison des statistiques portant sur le contenu des deux textes montre un usage plus fréquent de verbes factifs, de pronoms personnels tels que «nous » et "vous», et l'absence de notions de doute dans le discours de Laurent Fabius. Les verbes factifs utilisés sont les suivants: rassembler, favoriser, utiliser, créer, démocratiser, simplifier, associer, réorienter, remplacer, moderniser, accroitre, conserver, former. Ces verbes correspondent à l'exposé d'un programme qui se justifie à lui seul, le registre verbal utilisé étant à la fois positif (favoriser, simplifier, associer, moderniser, accroitre) et rassurant (démocratiser, réorienter, remplacer, conserver), en s'appuyant implicitement sur la légitimité de la parole gouvernementale (rassembler). L'ensemble de ces verbes sont aussi à même de traduire l'action et l'implication à la fois du ministre (" je») et celle du gouvernement (au travers de l'usage ambigu, parce qu'inclusif, du «nous»). S'agissant, plus particulièrement, de l'emploi du «vous», il intervient uniquement à un instant très précis, dans les toutes dernières phrases du discours. Dès lors, le ministre fait appel très clairement au soutien des membres de Fondact et de son président, et plus généralement à l'assistance, afin de soutenir la mise en application du projet de loi. Cette démarche correspond, de la sorte, à l'image de l'association Fondact conçue non seulement 
comme un lieu d'échange entre les pouvoirs publics et les différents acteurs de l'entreprise, mais également comme un lieu privilégié d’influence.

\begin{tabular}{|l|r|r|r|r|}
\cline { 2 - 5 } \multicolumn{1}{c|}{} & \multicolumn{2}{c|}{ Texte de Laurent Fabius } & \multicolumn{2}{c|}{ Texte de Christian Poncelet } \\
\hline Catégorie & Nombre & \multicolumn{1}{c|}{ Taux } & Nombre & \multicolumn{1}{c|}{ Taux } \\
\hline Verbe & 118 & $100,0 \%$ & 319 & $100,0 \%$ \\
Factif & 69 & $58,5 \%$ & 129 & $40,4 \%$ \\
Statif & 28 & $23,7 \%$ & 116 & $36,4 \%$ \\
Déclaratif & 21 & $17,8 \%$ & 72 & $22,6 \%$ \\
Performatif & 0 & $0,0 \%$ & 2 & $0,6 \%$ \\
\hline Modalisation & 44 & $100,0 \%$ & 141 & $100,0 \%$ \\
$\ldots$ & & & & \\
Doute & 0 & $0,0 \%$ & 3 & $2,1 \%$ \\
Négation & 6 & $13,6 \%$ & 29 & $20,6 \%$ \\
\hline Pronom & & & & \\
Personnel & 20 & $100,0 \%$ & 104 & $100,0 \%$ \\
«Je » & 6 & $30,0 \%$ & 21 & $20,2 \%$ \\
«ll» & 6 & $30,0 \%$ & 35 & $33,7 \%$ \\
« Nous » & 3 & $15,0 \%$ & 7 & $6,7 \%$ \\
«Vous " & 2 & $10,0 \%$ & 8 & $7,7 \%$ \\
«lls " & 0 & $0,0 \%$ & 8 & $7,7 \%$ \\
«On » & 1 & $5,0 \%$ & 10 & $9,6 \%$ \\
\hline
\end{tabular}

Tableau 3. Résumé des statistiques catégorielles des deux textes

Cette dernière dimension n'échappe probablement pas à la clairvoyance de Christian Poncelet. Il développe, en effet, une argumentation basée sur une majorité de verbes statifs ${ }^{12}$ et déclaratifs, avec cependant une fréquence plus forte de négations ${ }^{13}$. À cela s'ajoutent la présence de notions de doute ${ }^{14}$, l'usage du pronom personnel « ils » et une moins forte implication personnelle (« je »). La présence de verbes déclaratifs tels que dire, répéter, souhaiter, connaitre, contredire, contester, rappeler, demander, entendre, croire, savoir, expliquer, refuser, préférer, prononcer, interroger, voir, révèle un discours d'opposition, axé sur un effort pédagogique de réflexion et d'explication, mettant en doute la démarche gouvernementale. Ces verbes permettent également d'exprimer une

12. On relève notamment les verbes statifs suivants: sembler, comprendre, être, rester, constituer, concerner, consister, prolonger, avoir, devenir.

13. Les négations ont notamment pour objet d'apporter un correctif aux présentations du gouvernement: « les choses ne sont pas claires », «il importe de ne pas mélanger les objectifs », « ce problème ne pourra pas se résoudre en augmentant les cotisations sociales », " ces incantations ne sont pas à la mesure des défis », « il ne faut pas sombrer dans l'excès inverse », «il ne faut pas y voir un signe de prudence ».

14. Les notions de doute détectées par le logiciel Tropes correspondent aux termes suivants: peutêtre, apparemment, soi-disant. 
force de proposition (dire, répéter, expliquer, préférer, prononcer), et d'illustrer la faculté d'écoute (entendre) et d'implication (interroger) de l'orateur et, par extension, de son camp. Le discours a pour mission de déconstruire la légitimité de la parole gouvernementale en critiquant l'approche socialiste. S'agissant de l'usage des pronoms personnels, à la différence de Laurent Fabius, le président du Sénat utilise le "vous » au début, au milieu et à la fin de son discours. De la sorte, Christian Poncelet gagne en proximité avec son auditoire. Cet effet est renforcé par une oralité plus marquée du discours, avec de nombreuses répétitions, plusieurs points d'exclamation ainsi qu'un registre plus dramatique avec l'emploi de termes tels que «monstres », « pitoyable », « tétanisé $»^{15}$. Alors que Laurent Fabius opte pour la présentation objective d'une action légitime, Christian Poncelet opte pour une expression de tribun appelant une réaction du public. Une différence notoire réside finalement dans l'utilisation du pronom «ils $»^{16}$, évoquant allusivement le groupe des socialistes, qualifiés de " partisans de la modernité » devenus « champions du conservatisme ».

\section{La structure du discours}

La prise en compte de la structure du discours permet de prendre en considération l'ordre des arguments, c'est-à-dire l'enchainement des idées exposées. La sélection des propositions remarquables (voir: présentation de la méthode d'analyse) et des rafales ${ }^{17}$ du récit, obtenue grâce au logiciel Tropes, permet d'identifier les temps forts et de mieux localiser l'utilisation de certaines notions clés dans le fil du discours. On peut ainsi restituer la dynamique du discours (en présentant l'enchainement des termes clés en rafales) ainsi que les épisodes de celui-ci, au sein desquels on trouve une série de termes associés.

Chacune des propositions remarquables ${ }^{18}$ présentées ci-après, correspond à des termes présents dans le graphe en rafales précédent.

15. Soit les passages suivants du discours de Christian Poncelet: « [...] elle accouche de monstres juridiques, tels que le pitoyable compromis sur les stock options dont personne n'est plus en mesure d'expliquer le régime fiscal... »; "Les hommes chargés de déterminer et de conduire l'action du pays sont tétanisés par l'action et la réforme..."

16. Soit le passage suivant du discours de Christian Poncelet: «Au lieu de favoriser les évolutions, ils les contrarient. Au lieu de les anticiper, ils les enregistrent. Pire, au lieu de favoriser les valeurs en lesquelles ils croient, ils les trahissent et font le lit de toutes les inégalités... » [Souligné par nous.]

17. Cette approche - l'analyse par rafales, appliquée aux substantifs - permet de restituer l'enchainement des références saillantes du texte. Il s'agit d'un traitement séquentiel, présenté dans P. Lafon, 1981, "Statistiques des localisations des formes d'un texte », Mots. Les langages du politique, $\mathrm{n}^{\circ} 2$, mars, et P. Lafon, 1983, Dépouillement et statistiques en lexicométrie, Paris, Slaktine-Champion. Ce traitement consiste à détecter statistiquement les formes qui ont tendance à être plus citées dans certaines parties du texte.

18. Ces propositions remarquables sont extraites du discours de Laurent Fabius, disponible sur internet à l'adresse suivante: http://trebucq.u-bordeaux4.fr/mots_1.htm 


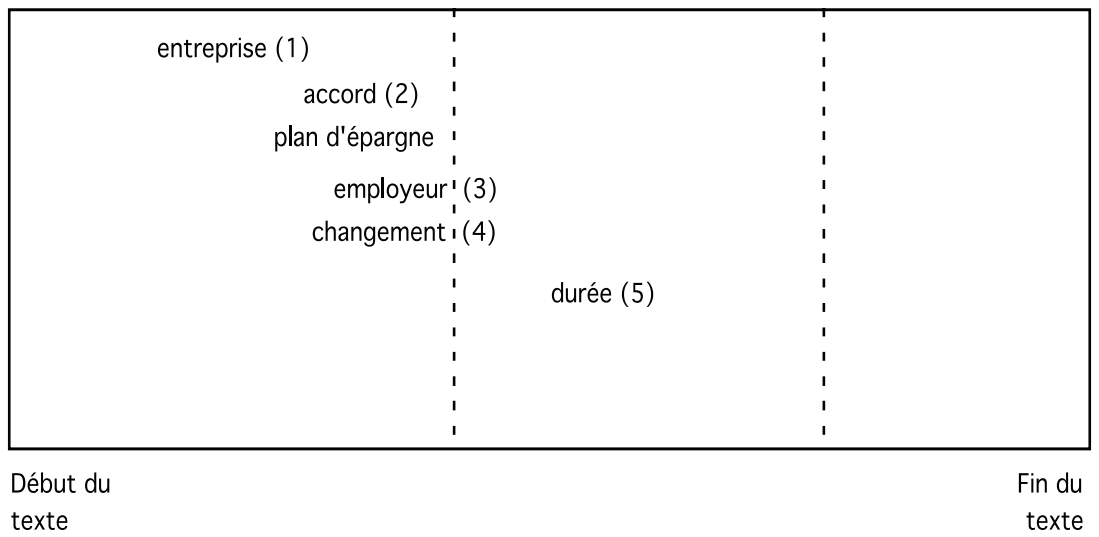

Graph. 3. Épisodes et rafales du discours de Laurent Fabius

(1) «Intéressement, participation, plan d'épargne d'entreprise étaient assez peu connus.»

(2) « Les entreprises de moins de 50 salariés qui auront conclu un accord de participation moins de 2 ans après la promulgation de la loi verront leur provision pour investissement portée de 25 à $50 \%$. »

(3) «Changements d'employeur et de plan d'épargne seront neutres au regard des droits du salarié. »

(4) «L'accélération du rythme du changement constitue un paramètre de la "nouvelle économie". »

(5) «Le PPESV (plan partenarial d'épargne salariale volontaire) sera donc aussi au service de l'économie solidaire. Obligeant à une négociation collective annuelle outre celle sur la rémunération, la durée et l'organisation du travail renforçant le rôle des salariés dans les conseils de surveillance des FCPE (fonds commun de placement d'entreprise). »

Chacune des propositions remarquables ${ }^{19}$ présentées ci-après, correspond à des termes présents dans le graphe en rafales précédent.

(1) « 38 sénateurs effectuent des stages d'entreprises pour la deuxième année consécutive. »

(2) « Deuxième interrogation, ce projet constitue-t-il un début de réponse au problème des retraites?»

(3) «Les "plans partenariaux d'épargne salariale volontaire” ressemblaient au départ comme des frères jumeaux aux “fonds partenariaux de retraite” imaginés par Laurent Fabius. »

19. Ces propositions remarquables sont extraites du discours de Christian Poncelet, disponible sur Internet à l'adresse suivante: http://trebucq.u-bordeaux4.fr/mots_1.htm 


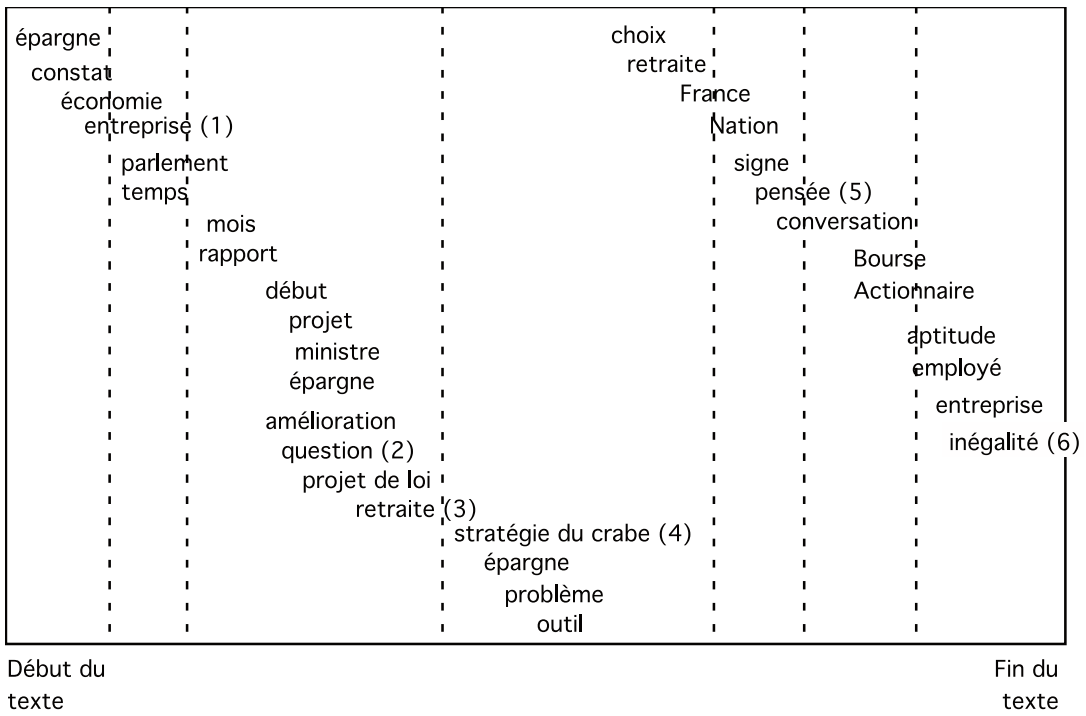

Graph. 4. Épisodes et rafales du discours de Christian Poncelet

(4) «Cette stratégie du crabe n'est pas la bonne.»

(5) «Notre pays est un pays gouverné par les idées.»

(6) « [ils] font le lit de toutes les inégalités; inégalités entre fonctionnaires et salariés [...]»

Les graphes en rafales permettent de confirmer la simplicité de la structure du discours de Laurent Fabius, avec une fin de discours marquée par l'absence de substantifs significatifs. En revanche, on dénombre sept épisodes dans le discours de Christian Poncelet, aboutissant finalement à un constat d'inégalité. À l'évidence, l'effort de présentation n'apparait pas à l'identique dans les deux cas. Le premier s'en tient à justifier la démarche retenue et à exposer l'essentiel des dispositions du projet de loi. En revanche, le second développe sa critique des orientations gouvernementales en mobilisant un ensemble plus complexe de références.

L'analyse, au travers du logiciel Tropes, des discours prononcés lors du forum Fondact par Laurent Fabius et par Christian Poncelet, met en évidence, d'une part, des conceptions très divergentes quant au mode de fonctionnement de la société, et d'autre part, la pérennité d'oppositions idéologiques très marquées à ce sujet.

L'analyse de contenu constitue un bon révélateur des différences d'opinion 
et de stratégie pouvant exister entre deux hommes politiques, idéologiquement opposés, à propos de l'épargne salariale. Laurent Fabius s'appuie sur une logique productiviste de l'actionnariat salarié pour étayer son projet de loi. Mais il se garde bien d'aborder la question tabou des fonds de pension, de même que celle du problème des retraites. Afin de conforter sa démarche, il souhaite obtenir l'appui et le soutien du mouvement Fondact.

En revanche, Christian Poncelet opte pour un style « offensif $»^{20}$. Bien qu'il rende hommage aux qualités intrinsèques de Laurent Fabius, qu'il considère comme un «homme intelligent »-assertion tendant à renforcer l'objectivité de son propos -, il n'en critique pas moins l'immobilisme du gouvernement refusant de mettre en place un dispositif susceptible de résoudre, à terme, grâce à l'actionnariat salarié, l'épineux problème des retraites. La question de l'actionnariat salarié continue de faire l'objet de sérieuses controverses entre les tenants des idéologies de droite et de gauche. Plus généralement, les motivations pouvant conduire à développer l'actionnariat salarié divergent largement. Or, l'organisation du débat politique sur ce sujet n'est pas sans incidences sur les réformes législatives, car celles-ci, à leur tour, ne manquent pas de transformer les rapports s'instaurant entre les divers acteurs de la gouvernance ${ }^{21}$ des entreprises.

Il n'est donc nullement fortuit qu'une question telle que la représentation des actionnaires salariés dans les conseils d'administration, ou bien celle du rôle d'un actionnariat salarié comme facteur de la stabilité du capital soient respectivement évoquées par Laurent Fabius et Christian Poncelet. Toutefois, force est de constater que le discours politique s'adresse le plus souvent - pour ne pas dire toujours - à des clientèles électorales. Aussi est-il intéressant de comprendre quel partenaire l'homme politique privilégie au sein du système de gouvernance des entreprises, et de fait, cela revient à poser la question de l'orientation donnée à l'actionnariat salarié. Celui-ci peut, tour à tour, servir les intérêts des salariés, des dirigeants, des clients et fournisseurs, des actionnaires, voire de la société en général.

Le discours de gauche, par la voix de Laurent Fabius, souhaite avant tout préserver, grâce à l'action étatique, l'intérêt des salariés. Le discours de droite, en l'occurrence celui de Christian Poncelet, semble en quête d'une stratégie médiane et consensuelle, ménageant à la fois l'intérêt des salariés et celui des dirigeants. Il développe une logique partenariale atténuant les conflits d'agence entre actionnaires, dirigeants et salariés grâce à un effacement des frontières

20. S. Milacic, 1999-2000, Introduction à l'analyse du discours politique, Bordeaux, IEP, Librairie Montaigne, p. 102.

21. La notion de gouvernance est utilisée à la fois en sciences de gestion et en science politique. Voir, respectivement: G. Charreaux éd., 1997, Le gouvernement des entreprises, Paris, Economica; G. Stoker, 1998, «Cinq propositions pour une théorie de la gouvernance », Revue internationale de sciences sociales, $\mathrm{n}^{\circ} 155$, mars, p. 19-30. 
entre ces statuts. On suppose que le salarié, une fois devenu actionnaire, n'opposera plus la même résistance au dirigeant. Pour autant, le règlement des conflits potentiels entre dirigeants et actionnaires reste loin d'être acquis.

Il s'agit en effet de savoir si l'actionnaire-salarié est en mesure de mieux délimiter les pouvoirs et les espaces discrétionnaires du dirigeant. L'enjeu du débat académique se trouve alors posé au niveau de la fonction objectif de la firme, c'est-à-dire en termes de création de valeur. Gérard Charreaux et Philippe Desbrières ${ }^{22}$ proposent la maximisation d'une valeur partenariale consistant à répondre non seulement aux exigences des actionnaires, mais également à celles des salariés et des autres «stakeholders» ou parties prenantes ${ }^{23}$. Cependant, selon Michael C. Jensen ${ }^{24}$, il ne peut y avoir d'autre fonction objectif pour la firme que la maximisation de la valeur actionnariale, sous peine de conduire à un gaspillage des ressources dommageable pour l'ensemble de la société capitaliste.

D'une certaine façon, on assiste à nouveau à un clivage entre deux conceptions de la gouvernance des entreprises: l'une, traditionnelle ${ }^{25}$, et fondée sur la valeur actionnariale, l'autre, plus progressiste ${ }^{26}$, et fondée sur la valeur partenariale. Il faut cependant souligner que si le dirigeant, le salarié, le client et le fournisseur deviennent actionnaires, alors la valeur actionnariale et la valeur partenariale tendent à se confondre. Dès lors se pose la question du partage de la richesse créée et, corrélativement, celle des rapports de force conduisant à définir les règles d'un tel partage. Ces règles sont régies par les contrats et la loi. Pour cette dernière composante, il semble justifié que la définition de telles règles soit soumise au débat démocratique.

22. G. Charreaux, P. Desbrières, 1998, «Gouvernance des entreprises: valeur partenariale contre valeur actionnariale », Revue finance contrôle stratégie, $n^{\circ}$ 2, juin, p. 57-88.

23. On notera que le projet de loi a retenu cette terminologie en qualifiant les plans d'épargne de "partenariaux». Ainsi, le projet de loi prévoit la mise en place d'un plan partenarial d'épargne salariale volontaire.

24. M. C. Jensen, 2000, "Value maximization, stakeholder theory, and the corporate objective function », forthcoming in Breaking the Code of Change, M. Beer et N. Norhia éd., Harvard Business School.

25. A. Shleifer, R.W. Vishny, 1997, "A survey of corporate governance », The Journal of Finance, vol. LII, $n^{\circ} 2$, juin.

26. R. E. Freeman, 1984, Strategic Management: A Stakeholder Approach, Pittman Books Limited. 